\title{
Pinolenic acid Mediated Anti-inflammatory and Immunometabolic Effects in Peripheral blood- derived Monocytes from patients with Rheumatoid Arthritis is Associated with Modulation of miRNA expression
}

Rabaa Takala ( $\square$ takalara@cardiff.ac.uk)

Cardiff University

Dipak Ramji

Cardiff University

Robert Andrews

Cardiff University

You Zhou

Cardiff University

Mustafa Farhat

Mohammed Elmajee

Shelley Rundle

Cardiff University

Ernest Choy

Cardiff University

\section{Research Article}

Keywords: rheumatoid arthritis, pro-inflammatory cytokines, purified monocytes, microRNAs, mRNA, polyunsaturated fatty acids, pinolenic acid, oxidative phosphorylation, pyruvate dehydrogenase kinase 4, single-immunoglobulin interleukin-1 receptor-related molecule

Posted Date: January 12th, 2022

DOI: https://doi.org/10.21203/rs.3.rs-1239491/v1

License: (c) (i) This work is licensed under a Creative Commons Attribution 4.0 International License. Read Full License 


\section{Abstract}

Objectives: Pinolenic acid (PNLA), an omega-6 polyunsaturated fatty acid from pine nuts, has antiinflammatory and anti-atherogenic effects. We aimed to investigate the actions of PNLA on activated purified monocytes from peripheral blood of patients with rheumatoid arthritis (RA).

Methods: Flow cytometry was used to assess the intracellular expression of TNF-a, IL-6, IL-1 $\beta$, and IL-8 in purified monocytes from patients with RA after lipopolysaccharide (LPS) stimulation with/without PNLA pre-treatment. The whole genomic transcriptomic (WGT) profile of PNLA-treated, and LPS-activated monocytes from patients with active RA was investigated by RNA-sequencing.

Results: PNLA reduced percentage of monocytes expressing the cytokines TNF-a by $23 \%(p=0.048)$, IL- 6 by $25 \%(p=0.011), I L-1 \beta$ by $23 \%(p=0.050)$ and IL- 8 by $19 \%(p=0.066)$. Canonical pathway analysis showed that PNLA inhibited oxidative phosphorylation $(p=9.14 \mathrm{E}-09)$ and mitochondrial dysfunction $(p=4.18 \mathrm{E}-08)$, while the sirtuin (SIRTs) signalling pathway was activated $(p=8.89 \mathrm{E}-06)$. Pathway analysis predicted upstream activation of peroxisome proliferator-activated receptors (PPARs), sirtuin3, and let7miRNA, which are anti-inflammatory and antioxidative. In contrast, DAP3, LIF and STAT3, which are involved in TNF-a, and IL- 6 signal transduction, were inhibited. Many miRNAs were modulated by PNLA suggesting potential post-transcriptional regulation of metabolic and immune response that has not been described previously. Multiple miRNAs target pyruvate dehydrogenase kinase-4 (PDK4), singleimmunoglobulin interleukin-1 receptor-related molecule (SIGIRR), mitochondrially encoded ATP synthase membrane subunit 6 (MT-ATP6) and acetyl-CoA acyltransferase 2 (ACAA2); genes implicated in cell metabolism, inflammation, and mitochondrial dysfunction.

Conclusion: PNLA has anti-inflammatory and immune-metabolic effects on monocytes that are pathogenic in RA and atherosclerosis. Dietary PNLA supplementation may regulate key miRNAs that are involved in mitochondrial, metabolic, and inflammatory pathways.

\section{Introduction}

RA is an autoimmune inflammatory disease that causes chronic joint inflammation. Cardiovascular diseases (CVDs) are the leading cause of death in patients with RA due to accelerated atherosclerosis. Monocytes/macrophages cause synovitis and produce pro-inflammatory cytokines. They are also important in atherosclerosis, as the most abundant immune cells within plaques and orchestrate the initiation, progression, and destabilisation of these lesions. Despite the increasing number of new treatments in RA, most patients do not achieve clinical remission, and residual pain and disability are common (1). Moreover, side effects and safety concerns of anti-rheumatic medications lead to poor adherence (2). A healthy and balanced diet including omega(n)-3 and other polyunsaturated fatty acid (PUFA) is recommended by American College of Rheumatology (ACR) and European League against Rheumatism (EULAR) to reduce the risk of RA. However, there is no recommendation on specific type of food due to limited scientific evidence. 
PNLA is an n-6 PUFA and initial studies showed anti-inflammatory and antioxidant actions in cell lines and animal models (3)(4)(5). Recent studies of PNLA on RA patients' peripheral blood (PB) samples identified several anti-atherogenic and anti-inflammatory effects (6). Takala et al in 2021 have demonstrated that PNLA significantly reduces monocyte migration, lipid uptake and macropinocytosis in THP-1 and primary cultures of macrophages in-vitro and ex-vivo(6). They also demonstrated that PNLA produces a significant reduction in the levels of TNF-a, IL-6, and PGE2 in supernatants of lipopolysaccharide (LPS) activated PBMCs from RA patients and healthy controls (HCs). Previous transcriptomic work on PBMCs found that PNLA regulates the expression of metabolic genes, including PDK4, FBP1, and SERPINE1. Moreover, bioinformatic analyses identified that PNLA inhibits NF-kB, STAT1, IL-1 and CCR2, while activates PPARs (6). These data suggest that PNLA has potential anti-inflammatory and metabolic effects on activated PBMCs from RA patients and HCs.

In the current study, we wanted to extend our previous findings and focus on the anti-inflammatory the immunomodulatory effects of PNLA on purified monocytes. First, the proportion of CD14 ${ }^{+}$monocytes expressing pro-inflammatory cytokines TNF-a, IL-6, IL- $\beta$, and IL-18 was assessed by flow cytometry. Second, whole genomic transcriptome (WGT) of PNLA treated LPS activated monocytes from RA patients with active disease was assessed.

\section{Methods}

\section{RA Patients recruitment and PBMCs isolation}

Participants at least 18 years old with RA ( $n=20$; mean age $=61.5$ years old) were recruited for assessing the intracellular levels of pro-inflammatory cytokines (TNF-a, IL-6, IL-1 $\beta$ and IL-8) expressed by purified monocytes after LPS-stimulation with or without PNLA. While for the transcriptome study, 8 ACPA positive RA patients with active disease (DAS28 score range: 4.8-5.7); (mean age=57 years old) and they were on biologic anti rheumatic drugs. All patients were recruited from the Rheumatology Department at the University Hospital of Wales, signed informed consent was obtained from all participants. The study was approved by the Research Ethics Committee for Wales 3 (reference no. 12/WA/0045). Detailed demographic and laboratory data are described in (Supplementary Tables S1, S2) for intracellular cytokines and WGT assessment, respectively. PBMCs were isolated by standard Ficoll density gradient centrifugation and methodological details are provided in (Supplementary Data S1).

\section{Negative selection and sorting of purified monocytes}

To enrich purified monocytes for assessment of intracellular cytokines using flow cytometry, monocytes were recovered by negative selection in cascades by magnetic-activated cell sorting (MACS) pan monocyte isolation kit (Miltenyi Biotec, UK) following the manufacturer's protocol. Pan monocyte isolation kit (Miltenyi Biotec, UK) uses a cocktail of biotinylated antibodies to select non-CD14 ${ }^{+}$cells, these cells are magnetically labelled using anti-biotin microbeads and separated from target CD14 ${ }^{+}$ monocytes cells using a magnetic column. $1 \times 10^{7}$ PBMCs were re-suspended in $100 \mu$ MACS (0.5\% BSA, 
$5 \mathrm{mM}$ EDTA, and $0.09 \%$ sodium azide in PBS), and $10 \mu \mathrm{l}$ of FcR blocking reagent and $10 \mu \mathrm{l}$ of biotinantibody cocktail (provided in the kit) were added. The suspension was then mixed and incubated for 10 min at $2-8^{\circ} \mathrm{C}$. A $20 \mu \mathrm{l}$ MACS buffer and $20 \mu \mathrm{l}$ of anti-biotin microbeads were added $/ 1 \times 10^{7}$ PBMCs. This suspension was mixed gently and incubated for another $10 \mathrm{~min}$ at $2-8^{\circ} \mathrm{C}$ before passing through a sterile magnetic column. The column was then washed with MACS buffer to ensure all purified monocytes were collected in a sterile $15 \mathrm{ml}$ Falcon tube. The flow through purified monocytes were centrifuged at $350 \mathrm{xg}$ for $5 \mathrm{~min}$ at room temperature. Number of viable cells were determined via trypan blue exclusion staining and counted using a haemocytometer and light microscope. Cells at a minimum density of $10^{6} \mathrm{cells} / \mathrm{ml}$ were cultured in complete RPMI medium at $37^{\circ} \mathrm{C}$ in $5 \% \mathrm{CO}_{2}$ controlled environment; the procedure was continued for cell surface/intracellular staining as described below or for the transcriptome analysis.

For the transcriptome study, the magnetically purified monocytes were passed through a second step of purification using FACS Aria III based cell sorting. In brief, cells were prepared for sorting, enriched monocyte cell suspensions were incubated with $2 \mu \mathrm{g} / \mathrm{ml}$ Fc human serum immunoglobulin (BD Biosciences; UK) at $4^{\circ} \mathrm{C}$ for 10 min to reduce non-specific binding by Fc receptors, and a small volume was aliquoted and labelled as unstained that was used for compensation. The cells were then stained with live/dead ${ }^{\text {TM }}$ (L/D) fixable viability stain (ThermoFisher Scientific, UK) with fluorochrome-conjugated antibodies to CD14 (BioLegend, USA) at a final dilution of 1/100. All experiments were controlled with appropriate isotype antibodies, compensation beads and unstained cells. To verify the purity and quantity of the sorted monocytes, purity check was performed under sterile conditions at $0-4^{\circ} \mathrm{C}$. The purity of the monocytes was $83.3 \%, 83 \%, 92.3 \%, 85.2 \%, 89.2 \%, 87 \%, 90.7 \%$, and $96 \%$ respectively for patients RA1RA8. Representative flow cytometry plots of the sorted monocytes population used in the study are shown in Supplementary Fig. S1.

\section{Monocyte treatment, cell surface and intracellular cytokines staining}

For assessment of intracellular cytokines by flow cytometry, enriched monocytes were seeded in 12-well plates at a minimum density of $10^{6}$ cells/well in $1 \mathrm{ml}$ of RPMl 1640 complete medium (RPMI 1640 medium containing $10 \%$ foetal calf serum (FCS), $100 \mathrm{U} / \mathrm{ml}$ penicillin, $100 \mathrm{mg} / \mathrm{ml}$ streptomycin and $2 \mathrm{mM}$ L-glutamine) along with 25 and $50 \mu \mathrm{M}$ PNLA (Cayman Chemicals, USA) or DMSO (Sigma-Aldrich, UK) vehicle control for 24 hour and some wells were stimulated with LPS $(100 \mathrm{ng} / \mathrm{ml})$ and Brefeldin (PFA; 10 $\mu \mathrm{g} / \mathrm{ml}$ ) both (Sigma-Aldrich, UK) for 8-9 hour or left un-stimulated as a control and the culture continued. Following all incubations, the monocytes were collected by detachment of the plastic wells (Supplementary data S2) and stained for surface fluorochrome-conjugated antibodies specific to monocyte surface receptors CD14, and CD16 (BioLegend, USA) as described in Supplementary data S2. The monocytes were then permeabilised using $0.3 \%$ saponin (Thermo Fisher Scientific, UK) diluted in PBS and stained for intracellular cytokines panel TNF-a, IL-1 $\beta$ (BioLegend, USA) and IL-6, IL-8 (BD Biosciences, UK) as in Supplementary data S2.

\section{Flow cytometry analysis}


A minimum of $10^{6}$ cells $/ \mathrm{mL}$ live cells/sample were collected using forward scatter (FSC) and side scatter (SSC) gating to avoid dead cells and debris and at least 50,000 events were acquired using flow cytometer (BD LSR-FORTESSA) in the 2-3-6-5 configuration (16 colour). Details on acquisition and analysis are provided in Supplementary data S2.

\section{Transcriptome analysis}

Sorted monocytes were washed with fresh RPMI medium, counted, and seeded in 6-well plates at a concentration of approximately $1 \times 10^{6}$ cells $/ \mathrm{mL}$ in RPMI 1640 complete medium along with either $25 \mathrm{mM}$ PNLA or DMSO vehicle control for 24 hours before stimulation with LPS $(100 \mathrm{ng} / \mathrm{ml})$ or vehicle control for 4 hours. The cell suspensions were then collected and washed with $\mathrm{Ca}^{2+}$ or $\mathrm{Mg}^{2+}$ free Dulbecco's PBS medium (Thermo Fisher Scientific, UK). The adherent cells were detached from the plastic by addition of $1 \mathrm{ml}$ accuatase cell dissociation reagent (Thermo Fisher Scientific, UK) pH 6.8/well, incubated for 5-8 min at $37^{\circ} \mathrm{C}$ with $5 \% \mathrm{CO}_{2}$, and then washed. The cell suspension was centrifuged at $400 \times \mathrm{g}$ at room temperature for $5 \mathrm{~min}$ and kept at $-80^{\circ} \mathrm{C}$ in $350 \mathrm{ml}$ buffer RLT (provided in RNeasy Mini kit) supplemented with $10 \%$ of 2-mercaptoethanol (Gibco Life Science, UK) as a lysate till RNA extraction.

\section{RNA extraction}

Total RNA was isolated using a RNeasy mini kit (Qiagen, Hilden, Germany) from purified monocytes following $100 \mathrm{ng} / \mathrm{ml}$ LPS and $25 \mu \mathrm{M}$ PNLA or vehicle treatment. RNA was purified using a RNeasy kit oncolumn with DNase I digestion (Qiagen) as described in Supplementary data S3. The cell lysates were stored at $-80^{\circ} \mathrm{C}$ and then passed through a series of spin columns to first bind genomic DNA, then RNA and finally to elute high-quality RNA. A quality control check for RNA was assessed as described in Supplementary data S3.

\section{Library construction, sequencing, and data processing}

\section{Library preparation including ribosomal RNA depletion}

In collaboration with the Wales Gene Park (Cardiff University), $5 \mathrm{ng}$ of total RNA was depleted of ribosomal RNA using the NEBNext ${ }^{\circledR}$ rRNA Depletion Kit (Human/Mouse/Rat), (New England BioLabs, (UK) Ltd). Ribosomal RNA depletion of each sample was assessed using the Agilent 4200 TapeStation with hsRNA ScreenTape (Agilent Technologies, Inc, UK).

The sequencing libraries were prepared using the $N E B \otimes{ }^{\circledR}$ Ultra ${ }^{T M}$ II Directional RNA Library Prep Kit for Illumina ${ }^{\circledR}$ (New England BioLabs, (UK) Ltd) protocol. The steps included RNA fragmentation and priming, $1^{\text {st }}$ strand cDNA synthesis, $2^{\text {nd }}$ strand cDNA synthesis, adenylation of $3^{\prime}$ ends, adapter ligation (adapter diluted 1:199) and PCR amplification (16-cycles). Following the addition of the PCR enrichment master mix, a unique index primer was added to each sample. The standard fragmentation procedure of $15 \mathrm{~min}$ at $94^{\circ} \mathrm{C}$ for samples for intact RNA $(>7)$ was reduced to 8 min at $94^{\circ} \mathrm{C}$ for partially degraded RNA with RIN values 2-6. Except for the replacement of RNAClean ${ }^{\circledR}$ XP beads and SPRI select beads by AMPure XP 
beads (Beckman Coulter $^{\circledR}$ ) the manufacturer's instructions were followed. The libraries were validated using Agilent 4200 TapeStation and high sensitivity DNA ScreenTapes (Agilent Technologies, Inc, UK) to ascertain the insert size, and the Qubit® (Thermo Fisher Technologies, UK) was used to perform the fluorometric quantitation.

\section{RNA-sequencing (RNA-seq)}

Following library quantification and validation, the normalized library pool was sequenced on one lane of an S1 (200 cycle) flow cell using XP workflow and 2x100 bp paired end dual indexed format on the NovaSeq6000 sequencing system (IIlumina ${ }^{\circledR}$ Inc, USA) according to the manufacturer's instructions. To conform to ENCODE guidelines, libraries were sequenced to have $>54$ million mapped reads (encodeproject.org/documents/cede0cbe-d324-4ce7-ace4-f0c3eddf5972).

\section{RNA-seq data processing and read mapping strategy}

Paired end reads from the Illumina sequencing were trimmed with Trim Galore (7) and assessed for quality using FastQC (8), using default parameters. Reads were mapped to the human GRCh38 reference genome using STAR software (9) and reads per gene were assigned using the featureCounts software (10) with the GRCh38.96 gene build gene transfer format (GTF). Both the reference genome and GTF were downloaded from the EnsembI FTP site (11). Duplicate reads were identified and marked using MarkDuplicates in Picard (Broad Institute).

\section{Normalisation and identification of differentially expressed genes (DEGs)}

Differential gene expression analyses used the DESeq2 package (12). Genes were discarded from the analysis if differential expression failed to be significant ( $p$ value $<0.05)(12)$. The initial WGT data were assessed with the web-based tool Morpheus (https://software.broadinstitute.org/morpheus/) using heatmaps to view changes in gene expression. The data were then separated into 3 groups; vehicle treated, vehicle treated/LPS stimulated, and PNLA treated/LPS stimulated monocytes for functional analysis including gene set enrichment analysis, gene ontology and further downstream pathway analysis.

\section{Heatmap of DEGs and principal component analysis (PCA)}

A heatmap and PCA are shown in (Supplementary Figs S2, S3). The heatmap was generated using broad Morpheus software and visualizations used fragments per kilobase per million mapped fragments (FPKM) reads and log2 fold change (log2 FC) comparing PNLA-treated and LPS-stimulated monocytes with those treated with vehicle and LPS from RA patients at equivalent time point (Supplementary Figs S2). Data sets were hierarchically clustered using 1 minus Pearson's correlation coefficient. PCA was performed in R using normalized data from the DESeq2 analysis, and data were clustered using the top 50 most DEGs. (Supplementary Fig S3). Each plot shows the results of the first two principal components. 


\section{Statistical analysis}

The statistics used were dependant on the experiments performed and depending on data distribution. Normality of data was tested using the Shapiro-Wilk test and confirmed with histograms and Q-Q plots in SPSS Statistics (version 23; IBM Corp., Armonk, NY, USA). FCS data files were analysed using FlowJo (version10.7, BD, UK) and the NoVo express. Percentage of cytokine expressing CD14 ${ }^{+}$monocytes was calculated using Microsoft Excel (version 16.52, Microsoft 365 Subscription, UK). Graphs and statistical analysis were performed using Graph Pad Prism (version 8 GraphPad Software, La Jolla, CA, USA). One way ANOVA was used to compare multiple groups followed by Dunn's post hoc analysis for pairwise comparisons. Spearman correlation was used to analyse the linear association using SPSS statistics (version 26; IBM Corp, Armonk, NY, USA) and 2 tailed Mann Whitney U test was applied to find the comparison between 2 groups. All values are presented as mean $\pm S E M, P$ - value $<0.05$ was considered significant for all the analysis.

\section{Results}

\section{Intracellular levels of several key cytokines were significantly reduced by PNLA}

The analysis was carried out on the enriched monocytes that constitute about $85-90 \%$ of all circulating blood monocytes, which we represent and refer to as $\mathrm{CD} 14^{+}$monocytes.

LPS stimulation increased CD14 ${ }^{+}$monocytes producing TNF-a $(20.6 \pm 2.96 \%)(p<0.0001)$ in comparison with non-stimulated monocytes $(3.69 \pm 0.83 \%)$. Pre-incubating monocytes with 25 or $50 \mu \mathrm{M}$ PNLA reduced the percentages of $\mathrm{CD} 14^{+}$cells producing TNF-a to $16.1 \pm 2.96 \%$ and $15.2 \pm 2.81 \%$ respectively $(p=0.048$ and 0.016 respectively) (Figure 1A).

Percentage of CD14 ${ }^{+}$monocytes expressing IL- 6 increased from $3.11 \pm 0.78 \%$ in non-stimulated cells to $17.9 \pm 2.49 \%(p<0.0001)$ after LPS stimulation. Pre-incubating monocytes with 25 or $50 \mu \mathrm{M}$ PNLA reduced the percentages of $\mathrm{CD} 14^{+}$cells expressing IL- 6 to $14.2 \pm 2.07 \%$ and $14.2 \pm 2.00 \%$ respectively ( $p=0.011$ and 0.013 respectively) (Figure 1B).

LPS stimulation increased CD14 ${ }^{+}$monocytes producing IL-1 $\beta$ to $42.99 \pm 4.94(p<0.0001)$ in comparison with non-stimulated monocytes $(9.86 \pm 2.91 \%)$. Pre-incubating monocytes with 25 or $50 \mu \mathrm{M}$ PNLA reduced the percentages of $\mathrm{CD} 14^{+}$cells expressing IL-1 $\beta$ to $37.1 \pm 3.62 \%$ and $37.0 \pm 3.61 \%$ respectively $(p=0.050$ and 0.049 respectively) (Figure 1C).

LPS stimulation increased percentage of CD14 ${ }^{+}$monocytes expressing IL-8 to $55.9 \pm 7.91 \%(p<0.001)$ in comparison with $4.81 \pm 3.8 \%$ for non-stimulated monocytes. Pre-incubating monocytes with 25 or $50 \mu \mathrm{M}$ PNLA slightly reduced the percentages of CD $14^{+}$cells expressing IL-8 to $47.2 \pm 7.97 \%$ and $48.2 \pm 6.58 \%$ respectively ( $p=0.0669$ and 0.0318 respectively) (Figure 1D). The differences were statistically significant only for $50 \mu \mathrm{M}$ PNLA. 


\section{Intracellular cytokines expression upon PNLA treatment did not significantly correlate with patients' demographic, clinical, and laboratory markers}

There was no statistically significant difference/correlation between percentage of reduction in CD14 ${ }^{+}$ monocytes expressing TNF-a, IL- 6 or IL-1 $\beta$ cytokines by PNLA against a variety of clinical indices and disease biomarkers after adjustment for multiple comparisons (Supplementary Table S3).

\section{Heatmap of DEGs and PCA}

A heatmap (Supplementary Fig. S2) showed the main clusters of DEGs of all treatment conditions mentioned. It can be visualised as two main upper and lower clusters. In the left upper cluster, the expression of vehicle treated monocyte genes were upregulated while PNLA and LPS treatment downregulated those genes on the right side. Conversely, in the lower cluster, the opposite pattern can be visualised. PCA results (Supplementary Fig. S3) also show samples clustering within groups demonstrating that inter-sample variation in gene expression is all within the normal range and not greater than the biology we hope to observe.

\section{Graphical summary and canonical pathways}

Graphical summary provides an overview of major biological themes in IPA (version: 33559992, Qiagen, USA); it selects the most significant entities in the core analysis and shows how they relate to each other. Biological process and regulators included have $z$-score $\geq 2$. Nodes are coloured by their activity predicted in the analysis where orange nodes are predicted to be activated with z-score $\geq 2$. In contrast, blue coloured entities with $z$ scores $\leq 2$ are predicted to be inhibited. As summary, PNLA treatment activated KLF15, AGO2, FLCN, SIRT3 and Sirtuin signalling pathways and inhibited STAT3, TFE3, CLPP and DAP3 (Figure 2A).

IPA was used to identify canonical pathways associated with differentially regulated transcripts identified between genotypes affected by PNLA and relevant pathways. They were determined by their p-value (Figure 2B). The canonical pathway most significantly associated with the differentially expressed transcripts was in 'oxidative phosphorylation' (p-value = 9.14E-09) (Supplementary Fig. S4). Of the 91 genes attributed to this pathway in the IPA reference dataset, 13 were differentially expressed in the experimental dataset which are ATP5PF, COX15, COX17, MT-ATP6, MT-C02, MT-ND1, MTND2, MTND4, MTND5, MT-ND4L, NDUFA7, NDUFA8, and NDUFB4. These proteins all located in the cytoplasm of the inner mitochondrial membrane and function as enzymes or transporters for the mitochondria.

Although oxidative phosphorylation is a vital part of metabolism, it produces reactive oxygen species (ROS) such as super oxide dismutase $\left(\mathrm{SOD}_{2}\right)$ and $\mathrm{H}_{2} \mathrm{O}_{2}$, which leads to propagation of free radicals, damaging cells and contributing to immune alterations and disease. Sirtuin signalling pathway was the third most significant canonical pathway that was activated in response to PNLA treatment versus LPS stimulated $(p=8.89 \mathrm{E}-06)$ after mitochondrial dysfunction $(p=4.18 \mathrm{E}-08)$. 


\section{Comparison analysis and upstream regulators}

Using IPA software, the comparison analysis was performed for all experimental conditions; vehicle, LPS stimulated, and LPS stimulated/ PNLA treated monocytes. In this analysis, we particularly sought at upstream analysis for the genes involved in the transcription of inflammatory cytokines, lipid metabolism and fatty acids actions, some drugs used for treating hyperlipidaemia and miRNAs. Data files were further exported for those genes from IPA and reproduced heatmaps using Broad Morpheus software. Heatmap visualisations using log2FC $>1.2$ are shown in Figure 3. Top predicted upstream regulators affected by PNLA treatment are shown in Supplementary Table S4.

\section{miRNAs impacted by LPS and PNLA}

Venn diagrams (Figure 4) showed that 35 miRNAs were differentially expressed in LPS stimulated versus unstimulated monocytes (7 were downregulated and 28 upregulated) (Figure 4A). PNLA treated/LPS stimulated versus unstimulated monocytes identified 68 miRNAs that were differentially expressed with 8 downregulated and 60 upregulated (Figure 4B). PNLA treatment/LPS stimulated versus LPS stimulated monocytes showed 6 downregulated miRNAs and 46 upregulated miRNAs (Figure 4C). List of these miRNAs are provided in Supplementary Table S5(A, B).

\section{miRNAs target mRNAs}

We determined mRNA targets of miRNA and the pathways involved in inflammation and cell metabolism by specific miRNA target prediction filter in IPA. This selected miRNAs-mRNA with a confidence of high or moderate prediction based on database.

Using miRNA target filter, top 72 significantly regulated miRNAs affected by PNLA treatment and LPS stimulations were uploaded into the core analysis, with 39 miRNAs having target information available. The associated mRNAs and pathways related are shown in Table 1. Expression fold change and expression $p$ value were determined based on calculated Fisher's exact test right tailed in IPA as shown.

Interestingly, one of mRNA targeted is PDK4, (Table 1) which is targeted by several miRNAs: miR-3173, miR-2861, miR-626, miR-28, miR-7150, miR-3188, miR-879-5p, miR-393-5p, miR-708-5p and miR-12118 (Figure 5A). This is consistent with our previous observation in PNLA treated PBMC (6).

SIGIRR is a mRNA that was actively targeted by miRNAs upon PNLA treatment. It negatively modulates immune responses because of its role in inhibition of NF-KB and TLRs signalling. SIGIRR is mostly expressed intracellularly in primary human monocytes, macrophages, and dendritic cells (DCs) (13) SIGIRR is targeted by 3 miRNAs as shown in Figure 5B, which are miR-7150, miR-1909-3p and miR-6868$5 p$ based on IPA database. SIGIRR downstream regulatory effects are shown in Figure 5C. Bioinformatic analyses also showed that the targets of these miRNAs are "metabolic mRNA" such as MT-ATP6, JMJD7PLA2G4B, ACAA2, ETFA, SPCS1 and GHRL, which are involved in metabolic signalling pathways, including, glucose metabolism, cholecystokinin (CCK) secretion, mitochondrial ATP synthase, NAD signalling pathway, oxidative phosphorylation and fatty acid $\beta$-oxidation. 


\section{Discussion}

Data from this study are the first to suggest that PNLA modulates immunometabolism, miRNAs of activated monocytes from patients with RA, and reduced the expression of intracellular inflammatory cytokines TNF-a, IL-6, IL- $\beta$, and IL-18.

Many DEGs from monocyte transcriptome, amongst these PDK4, ACADVL, CPT1A and SLC25A20 (Supplementary Table S6 (A)), were also identified previously in PBMCs from RA patients treated with PNLA (6). However, new transcription factors and biologic pathways were identified amongst these SIRTs was activated by PNLA. SIRTs signalling pathways were suggested as therapeutic targets in RA and atherosclerosis (14)(17). SIRT1 was found to be upregulated in synovial tissues and cells from patients with RA compared to OA (16). SIRT1, a NAD ${ }^{+}$dependent histone deacetylase, down regulates both the innate and adaptive immune response in mice. SIRT1 can inhibit NF-KB pathway directly by itself or indirectly via forkhead family of transcription factors (FoxO)(17)(25). SIRT1 deacetylase was initially shown to deacetylate RelA/p65 at lysine 310 residue, which leads to reduced NF-KB transcriptional activity (18)(19)(20). Substrates of SIRT1 are particularly abundant and include NF-KB RelA/p65, AP-1 family transcription factor c-Jun, and c-Myc. It was also reported that SIRT1 suppressed IL-12 production in human DCs through a direct interaction with the NF-KB transcription factor c-Rel (14). This may regulate NF-KB, which regulates the expression of cytokines, chemokines, and other pro-inflammatory agents. Also, recent studies suggest that SIRT1 has functions in chondrocytes and synoviocytes during inflammatory arthritis and modulates a variety of cell types during arthritis (19)(21)(22)(23). Overexpression of an enzymatically inactive form of SIRT1 reduced LPS-induced levels of TNFa in monocytes (21).

SIRTs have the potential to reduce the inflammatory component of atherosclerosis and may have the potential to alter the course of atherogenesis (24) (Supplementary Fig. S5). SIRT1 is highly expressed in human vascular endothelial cells (ECs) and regulates many cellular processes essential for cell survival, apoptosis, inflammation, stress resistance, cell growth, cell senescence and metabolism(24). SIRT1 deficiency contributes to increased inflammation, oxidative stress, foam cell formation, and impaired NO production and autophagy, thereby promoting vascular atherosclerosis and aging (25)(26) (27).

SIRT3 regulates several mitochondrial functions and has important roles in maintaining homeostasis. SIRT3 is believed to be a positive regulator of macroautophagy in adipocytes. In mature adipocytes, overexpression of SIRT3 activated macroautophagy, mainly on lipid droplets (LDs), through activating the AMP-activated protein kinase-Unc-51 like autophagy activating kinase 1 pathway, which in turn results in smaller LD size and reduced lipid accumulation. Moreover, SIRT3 overexpression causes instability of LDs in adipocytes and participates in the control of FA metabolism. SIRT3 knockout mice demonstrate abnormal lipid metabolism associated with abnormal accumulation of triglycerides in the livers of these animals during fasting (24). 
STAT3 and TFE3 are transcription factors that were inhibited by PNLA. STAT3 is an established target in RA as inhibitors of JAK and IL- 6 are approved treatment. TFE3 and TFEB are transcriptional regulators important in the activation of macrophages (28). Mice deficient of either TFEB or TFE3 showed reduced expression of cytokines. TFEB and TFE3 are master regulators of macroautophagy/autophagy and lysosome function in activated macrophages, and raises the possibility that these transcription factors may be of central importance in linking autophagy and lysosome dysfunction with inflammatory disorders, and the transcription of pro-inflammatory cytokines such as TNF- $\alpha$ and IL-1 $\beta$ requires the presence of both TFEB and TFE3 (29).

DAP3 is a mitochondrial ribosomal small subunit protein that is involved in mitochondrial physiology, apoptosis and TNF associated cell death pathways (30). Overexpression of DAP3 leads to a significant increase in cell death. DAP3 appears to act downstream of pro-apoptotic stimuli such as IFN- $\gamma$, TNF-a and Fas ligand, and is upstream to several caspases, such as caspase 8 and 9 (30).

To the authors best knowledge, this is the first study to show that many miRNAs were modulated by PNLA. Amongst these, let7 is a family of miRNAs that has variety of anti-inflammatory and antiatherogenic actions (15)(31)(32). Of note, several miRNA targeted activities of mRNA including PDK4 and SIGIRR. PDK4 regulates TNF-a and NF-KB (6)(33) whereas SIGIRR downstream regulatory effects include IL-1 $\beta$, TNF- $\alpha$, NF-KB and TRIM1 signalling pathways. SIGIRR was first characterised as an inhibitor of IL$1 R$ and TLR signalling by interaction with IRAK-1, and TRAF-6 in 293 human cell line (13). SIGIRR overexpression inhibited TLR-induced TNF-a, IL-10 and IFN-y-inducible protein 10 (IP-10) production in HMDMs and DCs. The role of SIGIRR as an inhibitor of inflammation was confirmed in vivo, since SIGIRR $(-/-)$ mice developed a more severe disease in CIA models (13). Overexpression of SIGIRR in primary human DCs as well as in M-CSF differentiated macrophages resulted in the inhibition of TLR-2/6, TLR-3, TLR-4, TLR-5, TLR-7/8, and IL-1R signalling (13). Overexpression of SIGIRR in human RA synovial cells led to the inhibition of spontaneously produced cytokines (13).

\section{Limitations and future work}

Results of mRNA and miRNA from transcriptomic study are exploratory and need to be confirmed by qPCR and/or western blot at protein level. In addition, the repressive effect of distinct miRNA species on their putative targets needs to be validated and confirmed by Luciferase assays. RA is a heterogenous disease and our study sample is small. Even though we did not find any association between disease features and the effect of PNLA on cytokine expression by monocytes, we cannot adequately address whether the transcriptome profiles may be influenced by clinical features as they are based on only 8 patients.

\section{Conclusion}

PNLA reduces the proportion of activated CD14 monocytes expressing TNFa, IL-6, IL-1 $\beta$ and IL-8 in active RA patients. PNLA regulates several miRNAs that targeted mRNA involved in regulation of mitochondrial pathway, and hence modulation the inflammatory and metabolic pathways. Although replication and 
validation are needed, results in this study are promising, and when considered in conjunction with previously demonstrated immunomodulatory effects of PNLA. Dietary supplement of PNLA may be beneficial for articular and vascular disease in patients with RA and atherosclerosis and may highlight the potential benefits of this PUFA as a potential anti-inflammatory agent.

\section{Abbreviations}

RA: Rheumatoid arthritis; PNLA: Pinolenic acid; PDK4: Pyruvate dehydrogenase kinase-4; miRNA: microRNA; ACR: American college of Rheumatology; EULAR: European league against Rheumatism; NFKB: Nuclear factor kappa; PGE2: prostaglandin E2; SIGIRR: Single-immunoglobulin interleukin-1 receptorrelated molecule; DAP3: Death associated protein-3; WGT: Whole genomic transcriptome; SD: Standard deviation; SEM: Standard error of the mean; EC: endothelial cell; DC: dendritic cell; FoxO: Forkhead family of transcription factors; SIRT: Sirtuin; TLRs: Tall like receptor; STAT: Signal transducer and activator of transcription ; PUFA: Polyunsaturated fatty acid; MACS: Magnetic activated cell sorting; DMSO: Dimethyl sulphoxide; CVDs; Cardiovascular diseases.

\section{Declarations}

\section{Acknowledgements}

The authors would like to thank all RA patients who participated in this study. We thank our colleagues Dr. Ismail Taban and James Burston for their technical assistance. We acknowledge our colleagues at Wales Gene Park (WGP) for their insight and expertise that assisted this research, for their technical support in generating the next generation sequencing data. WGP is an infrastructure support group funded by Welsh Government by Health and Care Research Wales. We acknowledge Versus Arthritis, Health and Care Research Wales CREATE Centre award grant number (20016) and Cardiff University for their support of this study.

\section{Funding}

This work was funded by a PhD studentship from the Libyan government grant number (513253).

\section{Authors contribution}

R.T., D.P.R. and E.C. were responsible for study conception, design, and data interpretation. E.C. was responsible for patient recruitment, sample, and clinical data collection. R.T. conducted laboratory experiments and the data analysis. R.T. prepared the manuscript and the figures. M.E. made critical review for intellectual content. R.A. did the basic bioinformatic work and Y.Z. designed the transcriptomic data analysis. M.F. assisted with flow cytometry and protocol design. S.R. was responsible for library construction and RNA sequencing. All authors critically revised and approved the final manuscript to be published. 


\section{Corresponding author}

Correspondence to Rabaa Takala

\section{Ethics approval and consent to participate}

The study was approved by the Research Ethics Committee for Wales 3 (reference no. 12/WA/0045). All patients signed a consent form approved by the local institutional review boards (University Hospital of Wales) before participating in this study.

\section{Consent for publication}

Yes.

\section{Competing interest}

The authors have declared no conflicts of interest.

\section{Availability of data and materials}

Data will be available upon reasonable request to the corresponding author by any qualified researchers who engage in independent scientific research and will be provided following review and approval of a research proposal and Statistical Analysis Plan (SAP) and execution of a Data Sharing Agreement (DSA). All data are incorporated into the article and its online supplementary material.

\section{Supplementary information}

Supplementary data are available at additional file (1).

\section{References}

1. Ferreira RJO, Dougados M, Kirwan JR et al. Drivers of patient global assessment in patients with rheumatoid arthritis who are close to remission: an analysis of 1588 patients. Rheumatology 2017;56:1573-8.

2. Smolen JS, Gladman D, McNeil HP et al. Predicting adherence to therapy in rheumatoid arthritis, psoriatic arthritis or ankylosing spondylitis: a large cross-sectional study. RMD Open 2019;5:e000585

3. Chen S-J, Chuang L-T, Liao J-S, Huang W-C, Lin H-H. Phospholipid incorporation of non-methyleneinterrupted fatty acids (NMIFA) in murine microglial BV-2 cells reduces pro-inflammatory mediator production. Inflammation. 2015;38(6):2133-45.

4. Chen S-J, Huang W-C, Shen H-J, Chen R-Y, Chang H, Ho Y-S, et al. Investigation of Modulatory Effect of Pinolenic Acid (PNA) on Inflammatory Responses in Human THP-1 Macrophage-Like Cell and Mouse Models. Inflammation [Internet]. 2020 Apr [cited 2020 Aug 30];43(2):518-31. 
5. Chuang L-T, Tsai P-J, Lee C-L, Huang Y-S. Uptake and incorporation of pinolenic acid reduces n-6 polyunsaturated fatty acid and downstream prostaglandin formation in murine macrophage. Lipids 2009;44:217-24.

6. Takala R, Ramji DP, Andrews R, Zhou Y, Burston J, Choy E. Anti-inflammatory and immunoregulatory effects of pinolenic acid in rheumatoid arthritis. Rheumatology [Internet]. 2021 Jun 3 [cited 2021 Jul 14]; (keab467).

7. Babraham Bioinformatics. Trim Galore. https://www.

bioinformatics.babraham.ac.uk/projects/trim_galore/ (1 September 2021, date last accessed).

8. Babraham Bioinformatics. FastQC. https://www. bioinformatics.babraham.ac.uk/projects/fastqc/ (1 September 2021, date last accessed).

9. Dobin A, Davis CA, Schlesinger F et al. STAR: ultrafast universal RNA-seq aligner. Bioinformatics 2013;29: 15-21.

10. Liao Y, Smyth GK, Shi W. featureCounts: an efficient general-purpose program for assigning sequence reads to genomic features. Bioinformatics 2014; 30:923-30.

11. Ensembl. http://www.ensembl.org/info/data/ftp/index. html/ (1 September 2021, date last accessed).

12. Love MI, Huber W, Anders S. Moderated estimation of fold change and dispersion for RNA-seq data with DESeq2. Genome Biol 2014;15:550.

13. Drexler SK, Kong P, Inglis J, Williams RO, Garlanda C, Mantovani A, et al. SIGIRR/TIR-8 is an inhibitor of toll-like receptor signalling in primary human cells and regulates inflammation in models of rheumatoid arthritis. Arthritis \& Rheumatism [Internet]. 2010 Aug 1 [cited 2021 June 22];62(8):224961.

14. Kong S, Yeung P, Fang D. The Class III Histone Deacetylase Sirtuin 1 in Immune Suppression and Its Therapeutic Potential in Rheumatoid Arthritis. Journal of Genetics and Genomics [Internet]. 2013 Jul 20;40(7):347-54.

15. Furer V, Greenberg JD, Attur M, Abramson SB, Pillinger MH. The role of microRNA in rheumatoid arthritis and other autoimmune diseases. [Internet]. $2010 \mathrm{Jul}$ 1;136(1):1-15.

16. Krause A, Scaletta N, Ji J-D, Ivashkiv LB. Rheumatoid Arthritis Synoviocyte Survival Is Dependent on Stat3. J Immunol [Internet]. 2002 Dec 1;169(11):6610.

17. 17. de Gregorio E, Colell A, Morales A, Marí M. Relevance of SIRT1-NF-KB axis as therapeutic target to ameliorate inflammation in liver disease. International Journal of Molecular Sciences. 2020;21(11):3858.

18. Qiu Y, Zhou X, Liu Y, Tan S, Li Y. The Role of Sirtuin-1 in Immune Response and Systemic Lupus Erythematosus. Frontiers in immunology. 2021;12.

19. Yeung F, Hoberg JE, Ramsey CS, Keller MD, Jones DR, Frye RA, et al. Modulation of NF-KB-dependent transcription and cell survival by the SIRT1 deacetylase. The EMBO journal. 2004;23(12):2369-80. 
20. Li G, Xia Z, Liu Y, Meng F, Wu X, Fang Y, et al. SIRT1 inhibits rheumatoid arthritis fibroblast-like synoviocyte aggressiveness and inflammatory response via suppressing NF-KB pathway. Biosci Rep [Internet]. 2018 Jun 21;38(3):BSR20180541.

21. Niederer F, Ospelt C, Brentano F, Hottiger MO, Gay RE, Gay S, et al. SIRT1 overexpression in the rheumatoid arthritis synovium contributes to proinflammatory cytokine production and apoptosis resistance. Ann Rheum Dis [Internet]. 2011 Oct 1;70(10):1866.

22. Huang W, Shang W, Wang $H$, Wu W, Hou S. Sirt1 overexpression protects murine osteoblasts against TNF-a-induced injury in vitro by suppressing the NF-KB signaling pathway. Acta Pharmacologica Sinica. 2012;33(5):668-74.

23. Moon M-H, Jeong J-K, Lee Y-J, Seol J-W, Jackson C, Park S-Y. SIRT1, a class III histone deacetylase, regulates TNF-a-induced inflammation in human chondrocytes. Osteoarthritis and cartilage. 2013;21(3):470-80.

24. Sosnowska B, Mazidi M, Penson P, Gluba-Brzózka A, Rysz J, Banach M. The sirtuin family members SIRT1, SIRT3 and SIRT6: Their role in vascular biology and atherogenesis. Atherosclerosis [Internet]. 2017 Oct 1;265:275-82.

25. Brunet A, Sweeney LB, Sturgill JF, Chua KF, Greer PL, Lin Y, et al. Stress-dependent regulation of FOXO transcription factors by the SIRT1 deacetylase. science. 2004;303(5666):2011-5.

26. Chung S, Yao H, Caito S, Hwang J, Arunachalam G, Rahman I. Regulation of SIRT1 in cellular functions: role of polyphenols. Archives of biochemistry and biophysics. 2010;501(1):79-90.

27. Zeng H, Fu Y, Yu W, Lin J, Zhou L, Liu L, et al. SIRT1 prevents atherosclerosis via liver-X-receptor and NF-KB signaling in a U937 cell model. Molecular Medicine Reports. 2013;8(1):23-8.

28. Brady OA, Martina JA, Puertollano R. Emerging roles for TFEB in the immune response and inflammation. null [Internet]. 2018 Feb 1;14(2):181-9.

29. Pastore N, Brady OA, Diab HI, Martina JA, Sun L, Huynh T, et al. TFEB and TFE3 cooperate in the regulation of the innate immune response in activated macrophages. Autophagy. 2016;12(8):124058.

30. Kim H-R, Chae H-J, Thomas M, Miyazaki T, Monosov A, Monosov E, et al. Mammalian dap3 is an essential gene required for mitochondrial homeostasis in vivo and contributing to the extrinsic pathway for apoptosis. The FASEB Journal [Internet]. 2007 Jan 1 [cited 2021 Jun 11];21(1):188-96.

31. Chen K-C, Juo S-HH. MicroRNAs in atherosclerosis. The Kaohsiung Journal of Medical Sciences. 2012 Dec 1;28(12):631-40.

32. Brennan E, Wang B, McClelland A, Mohan M, Marai M, Beuscart $O$, et al. Protective effect of let-7 miRNA family in regulating inflammation in diabetes-associated atherosclerosis. Diabetes. 2017;66(8):2266-77.

33. Dlamini Z, Ntlabati P, Mbita Z, Shoba-Zikhali L. Pyruvate dehydrogenase kinase 4 (PDK4) could be involved in a regulatory role in apoptosis and a link between apoptosis and insulin resistance. Experimental and Molecular Pathology. 2015 Jun 1;98(3):574-84. 


\section{Tables}

Table. 1. Selected miRNAs target mRNA and the relevant associated pathways as per IPA database. 


\begin{tabular}{|c|c|c|c|c|c|c|}
\hline miRNA ID & $\begin{array}{l}\text { Expr p- } \\
\text { value }\end{array}$ & $\begin{array}{l}\text { Expr } \\
\text { Log } \\
\text { Ratio }\end{array}$ & $\begin{array}{l}\text { mRNA } \\
\text { symbol }\end{array}$ & $\begin{array}{l}\text { Expr p- } \\
\text { value }\end{array}$ & $\begin{array}{l}\text { Expr } \\
\text { Log } \\
\text { Ratio }\end{array}$ & Pathway \\
\hline miR-4521 & 0.0542 & 2.853 & \multirow[t]{3}{*}{ ACAA2 } & \multirow[t]{3}{*}{0.00344} & \multirow[t]{3}{*}{0.91} & \multirow{3}{*}{$\begin{array}{l}\text { Fatty acid } \beta \text {-oxidation I, } \\
\text { Glutaryl-CoA degradation, I, } \\
\text { ketogenesis, ketolysis, super } \\
\text { pathway of cholesterol } \\
\text { biosynthesis. }\end{array}$} \\
\hline miR-7150 & 0.0429 & 1.805 & & & & \\
\hline miR-8066 & 0.0423 & 3.642 & & & & \\
\hline miR-4440 & 0.0136 & 2.997 & ATMIN & 0.0304 & -0.243 & $\begin{array}{l}\text { Role of CHK Proteins in cell } \\
\text { cycle checkpoint control }\end{array}$ \\
\hline miR-3173 & 0.0011 & 1.975 & CRABP2 & 0.0407 & 1.488 & $\begin{array}{l}\text { Acute phase response, retinoid } \\
\text { acid mediated apoptosis } \\
\text { signalling }\end{array}$ \\
\hline miR-374B & 0.0034 & 1.382 & ETFA & 0.0498 & 0.248 & NAD Signalling Pathway \\
\hline miR-1909 & 0.0303 & -0.548 & FZD2 & 0.0334 & -1.057 & $\begin{array}{l}\text { Adipogenesis pathway, } \\
\text { osteoarthritis pathway, role of } \\
\text { macrophages, fibroblasts and } \\
\text { Endothelial Cells in rheumatoid } \\
\text { arthritis, role of osteoblasts, } \\
\text { osteoclasts and chondrocytes } \\
\text { in rheumatoid arthritis. }\end{array}$ \\
\hline $\operatorname{miR}-4440$ & 0.0136 & 2.997 & \multirow[t]{2}{*}{ GHRL } & \multirow[t]{2}{*}{0.0388} & \multirow[t]{2}{*}{0.429} & \multirow[t]{2}{*}{ Leptin Signalling in Obesity } \\
\hline miR-548L & 0.0712 & 1.677 & & & & \\
\hline miR1260B & 0.0206 & 0.901 & \multirow[t]{2}{*}{$\begin{array}{l}\text { JMJD7- } \\
\text { PLA2G4B }\end{array}$} & \multirow[t]{2}{*}{0.05} & \multirow[t]{2}{*}{0.483} & \multirow{2}{*}{$\begin{array}{l}\text { ERK/MAPK Signalling, } \\
\text { Glucocorticoid Receptor } \\
\text { Signalling, MIF Regulation of } \\
\text { Innate Immunity, MIF-mediated } \\
\text { Glucocorticoid Regulation, } \\
\text { Pathway, p38 MAPK Signalling, } \\
\text { Phospholipase C Signalling, } \\
\text { VEGF Family Ligand-Receptor } \\
\text { Interactions, CCR3 signalling in } \\
\text { eosinophils. }\end{array}$} \\
\hline miR-646 & 0.0702 & 3.95 & & & & \\
\hline miR-2861 & 0.0611 & -1.178 & LSM1 & 0.0312 & -0.282 & $\begin{array}{l}\text { Systemic lupus erythematosus } \\
\text { signalling }\end{array}$ \\
\hline miR-3934 & 0.0758 & 1.072 & \multirow[t]{3}{*}{ MT-ATP6 } & \multirow[t]{3}{*}{0.0307} & \multirow[t]{3}{*}{-0.991} & \multirow{3}{*}{$\begin{array}{l}\text { Mitochondrial dysfunction, } \\
\text { oxidative phosphorylation, } \\
\text { sirtuin signalling pathway, } \\
\text { estrogen receptor signalling, } \\
\text { glucocorticoid receptor } \\
\text { signalling. }\end{array}$} \\
\hline miR-671 & 0.0386 & -0.35 & & & & \\
\hline miR-3188 & 0.0358 & 1.958 & & & & \\
\hline
\end{tabular}




\begin{tabular}{|c|c|c|c|c|c|c|}
\hline miR-7150 & 0.0429 & 1.805 & PAIP1 & 0.0265 & -0.209 & $\begin{array}{l}\text { CSDE1 signalling pathway, EIF2 } \\
\text { signalling, Insulin secretion } \\
\text { signalling pathway. }\end{array}$ \\
\hline miR-3173 & 0.0611 & 1.975 & \multirow[t]{7}{*}{ PDK4 } & \multirow[t]{7}{*}{0.000364} & \multirow[t]{7}{*}{3.19} & \multirow{7}{*}{$\begin{array}{l}\text { Glucocorticoid receptor } \\
\text { signalling. Reelin signalling, } \\
\text { senescence pathway }\end{array}$} \\
\hline miR-2861 & 0.0611 & -1.178 & & & & \\
\hline${ }_{626}^{\mathrm{miR}-}$ & 0.0446 & 1.486 & & & & \\
\hline miR-28 & 0.0429 & 2.181 & & & & \\
\hline miR-7150 & 0.0358 & 1.805 & & & & \\
\hline miR-3188 & 0.0470 & 1.958 & & & & \\
\hline miR-3934 & 0.0611 & 1.072 & & & & \\
\hline miR-1909 & 0.0303 & -0.548 & \multirow[t]{3}{*}{ SIGIRR } & \multirow[t]{3}{*}{0.0445} & \multirow[t]{3}{*}{1.124} & \multirow{3}{*}{$\begin{array}{l}\text { NF-KB Signalling, TLR } \\
\text { signalling, TREM } 1 \text { signalling }\end{array}$} \\
\hline miR-7150 & 0.0429 & 1.805 & & & & \\
\hline $\begin{array}{l}\operatorname{miR}_{5 P}-6868- \\
-\end{array}$ & 0.0025 & 1.501 & & & & \\
\hline miR-637 & 0.0063 & -1.747 & SPCS1 & 0.00457 & -0.389 & $\begin{array}{l}\text { Insulin secretion signalling } \\
\text { pathway }\end{array}$ \\
\hline
\end{tabular}

\section{Supplementary}

Supplementary Tables S1-S6 are not available with this version

\section{Figures}


A

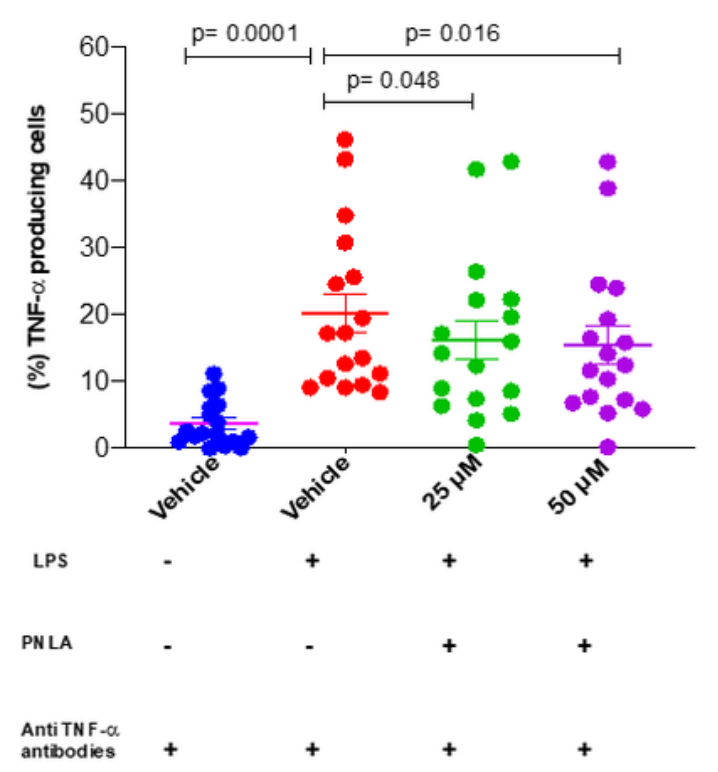

C

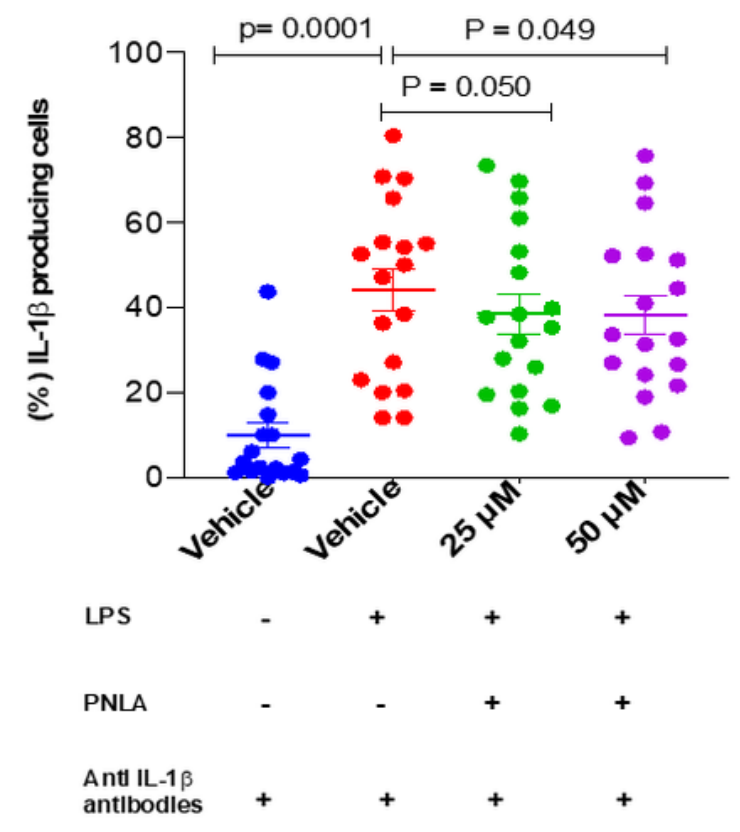

B
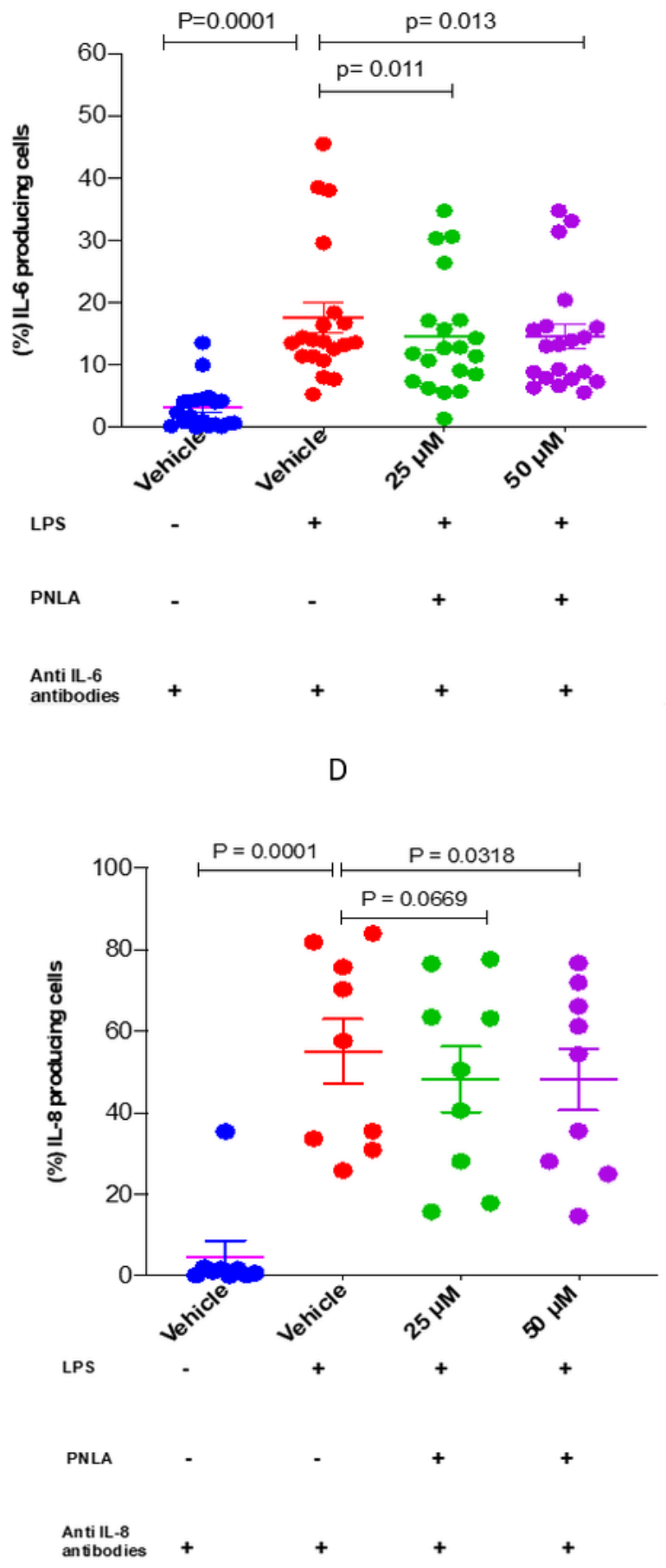

Figure 1

$\mathrm{CD}_{14}^{+}$monocytes expressing inflammatory cytokines were reduced significantly following PNLA treatment. TNF- $a$, IL-6 IL-1 $\beta$ and IL-8 were reduced (A), (B), (C) and (D) respectively. Purified monocytes were incubated with 25 or $50 \mu \mathrm{M}$ PNLA for 24-hours followed by $100 \mathrm{ng} / \mathrm{ml}$ LPS stimulation for 9 hours. Then, cells were stained and analysed for the expression of TNF-a, IL-6, IL-1 $\beta$ and IL-8 antibodies using flow cytometry; all samples run in duplicates or triplicates. The data are presented as mean +/- SEM, each 
dot $(\cdot)$ represents the average of one participant. Statistical analysis was performed using one-way ANOVA and a Dunn post-hoc-test. TNF-a, IL-6, IL-1 $\beta$ were assessed in ( $n=20)$, and IL-8 in ( $n=9)$ RA patients.

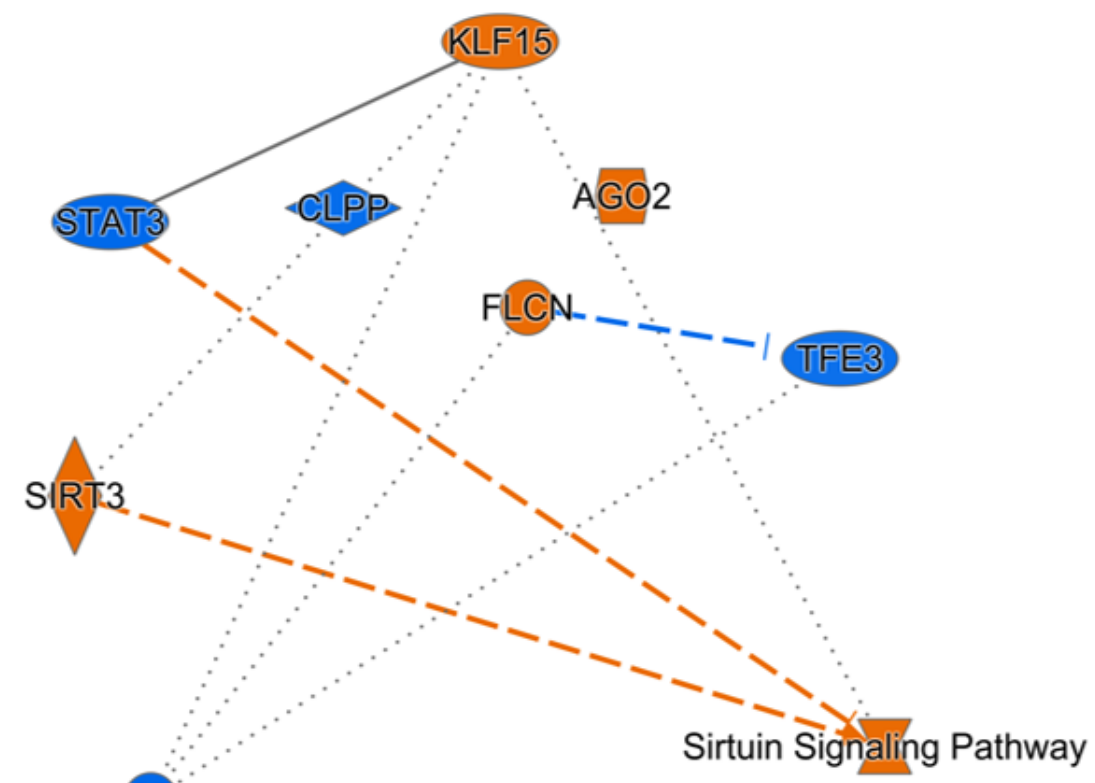

DAP3

A. Graphical summary

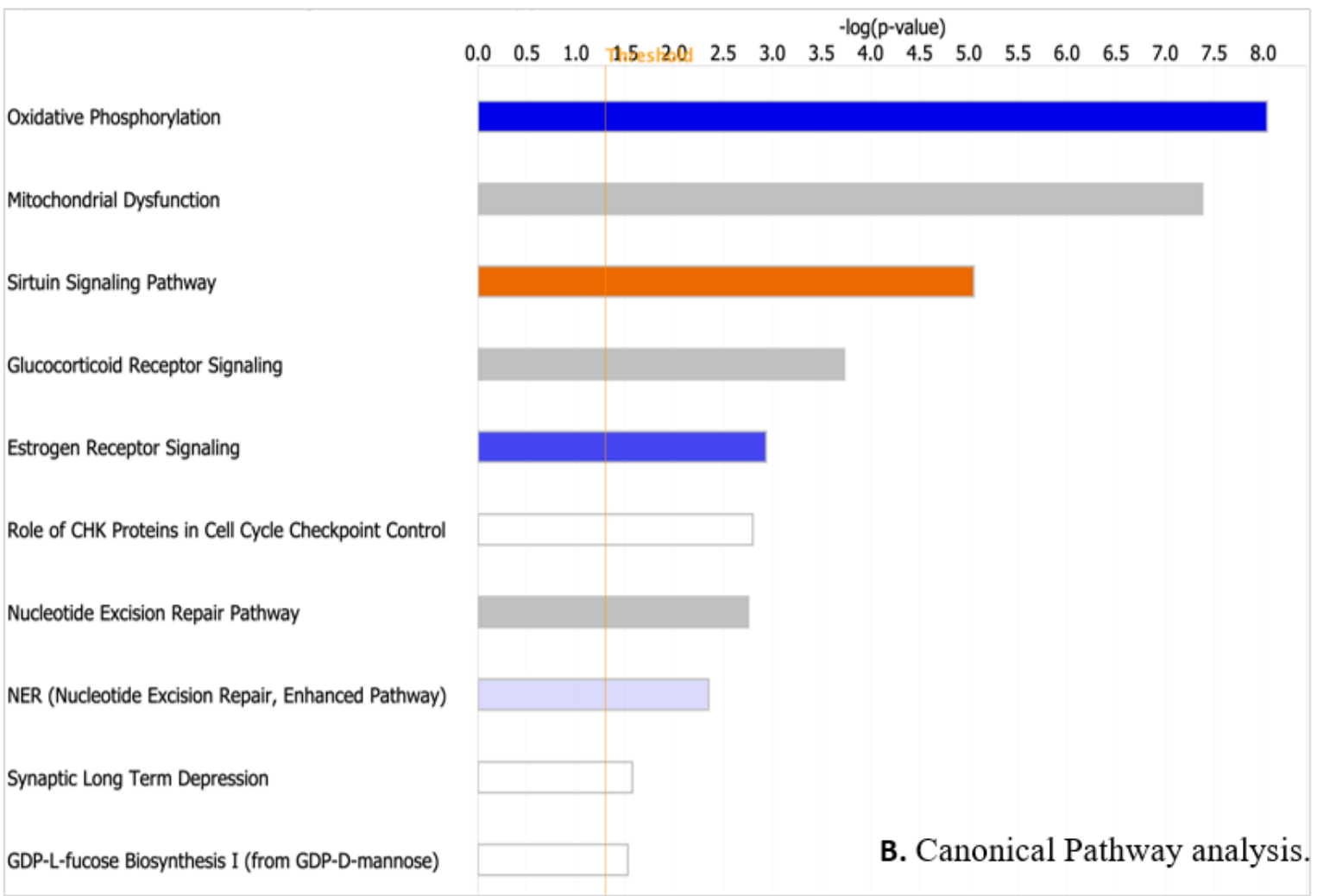

Figure 2 
Graphical summary and canonical pathway analysis. (A) Graphical summary showing the most significant transcription factors and regulators affected by PNLA. The orange colour goes with the direction of activation while the blue is the inhibition state. (B) Canonical pathways bar chart for PNLA effect on activated monocytes. The top 10 most significant pathways are shown, as calculated by Fisher's exact test right tailed. The colour of the bar is a measure of z-score which reflects the direction of the expression change (pathway activation or inhibition). An orange bar represents an overall positive zscore (majority of genes are upregulated) while a blue bar represents an overall negative z-score (majority of genes are downregulated). White bars possess a z-score which is zero or close to zero. Grey bars highlight pathways for which IPA is unable to make a prediction. The orange line graph represents the ratio (the number of genes that meet cut-off criteria in the dataset, divided by the total number of known genes attributed to that pathway in the IPA reference gene set). 


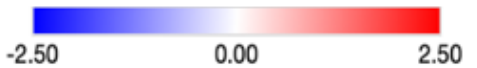

$$
\begin{aligned}
& \text { 웅 융 } \\
& \text { 业 } \\
& \text { 輨 }
\end{aligned}
$$$$
\text { id } \dot{\sim} \text { ' }
$$

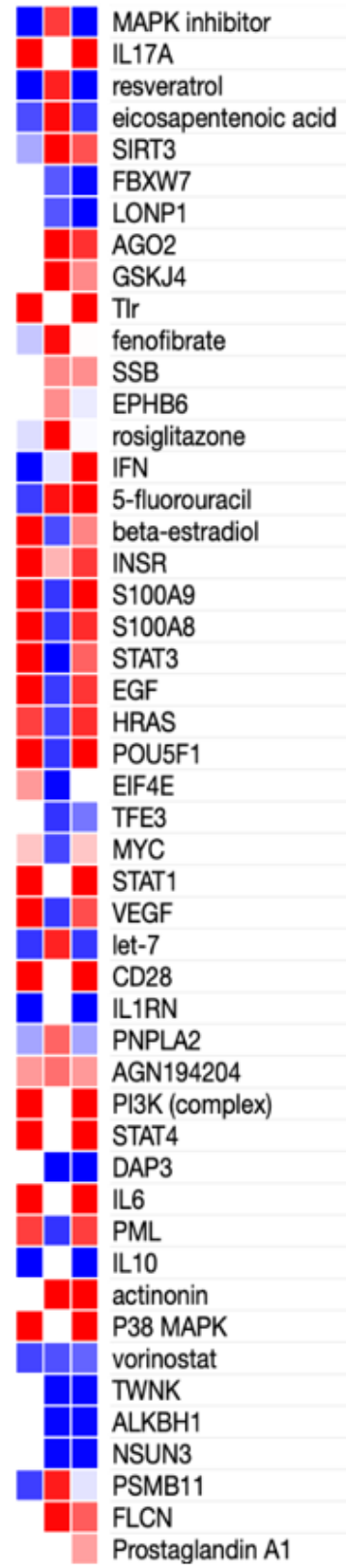

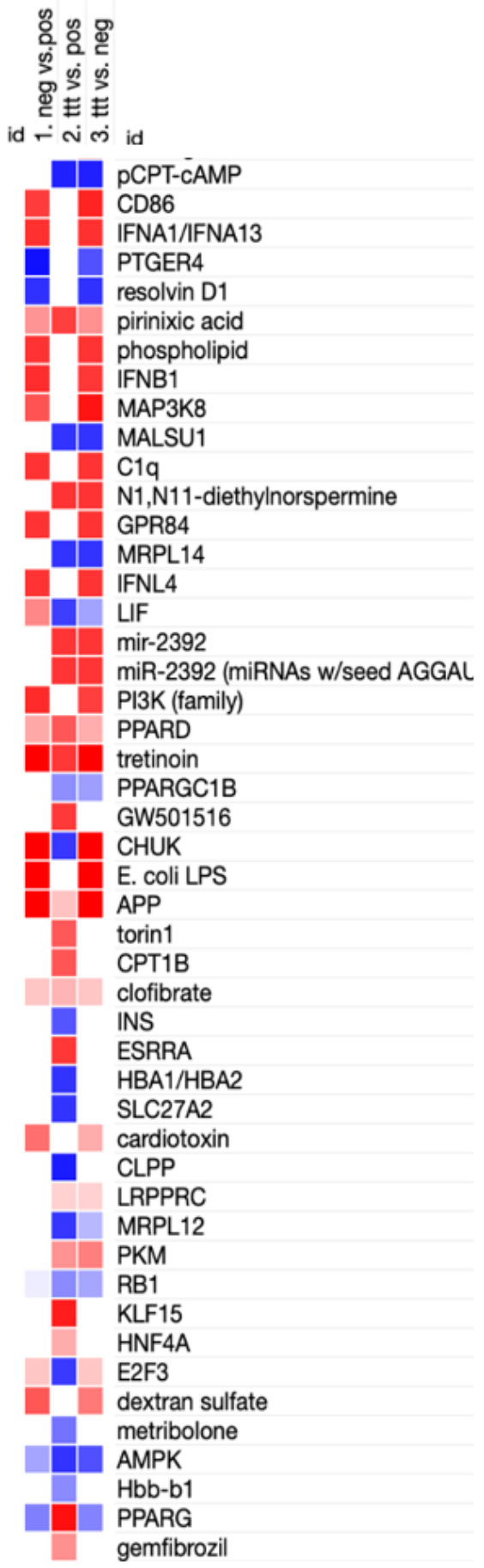

\section{Figure 3}

Heatmap of differentially expressed upstream regulators for comparison of RA patients. IPA analysis of DEGs associated with upstream regulators that predicted activated state (red) and predicted inhibited state (blue) are shown. Relative expression heat maps of the differentially expressed regulators regulated by; (1) unstimulated (vehicle) vs LPS-stimulated monocytes (2) PNLA-treated LPS-stimulated vs vehicletreated LPS-stimulated monocytes, and (3) PNLA-treated LPS-stimulated vs un-stimulated (vehicle- 
treated) monocytes (Log2 FC>1.2, $\mathrm{P}<0.05)$ are shown. The regulators shown here are mainly involved in cytokine production, lipid and fatty acid metabolism, drugs used for treating hyperlipidaemia, miRNAs and transcription factors. LPS: lipopolysaccharide; PNLA: pinolenic acid; ttt: treatment.

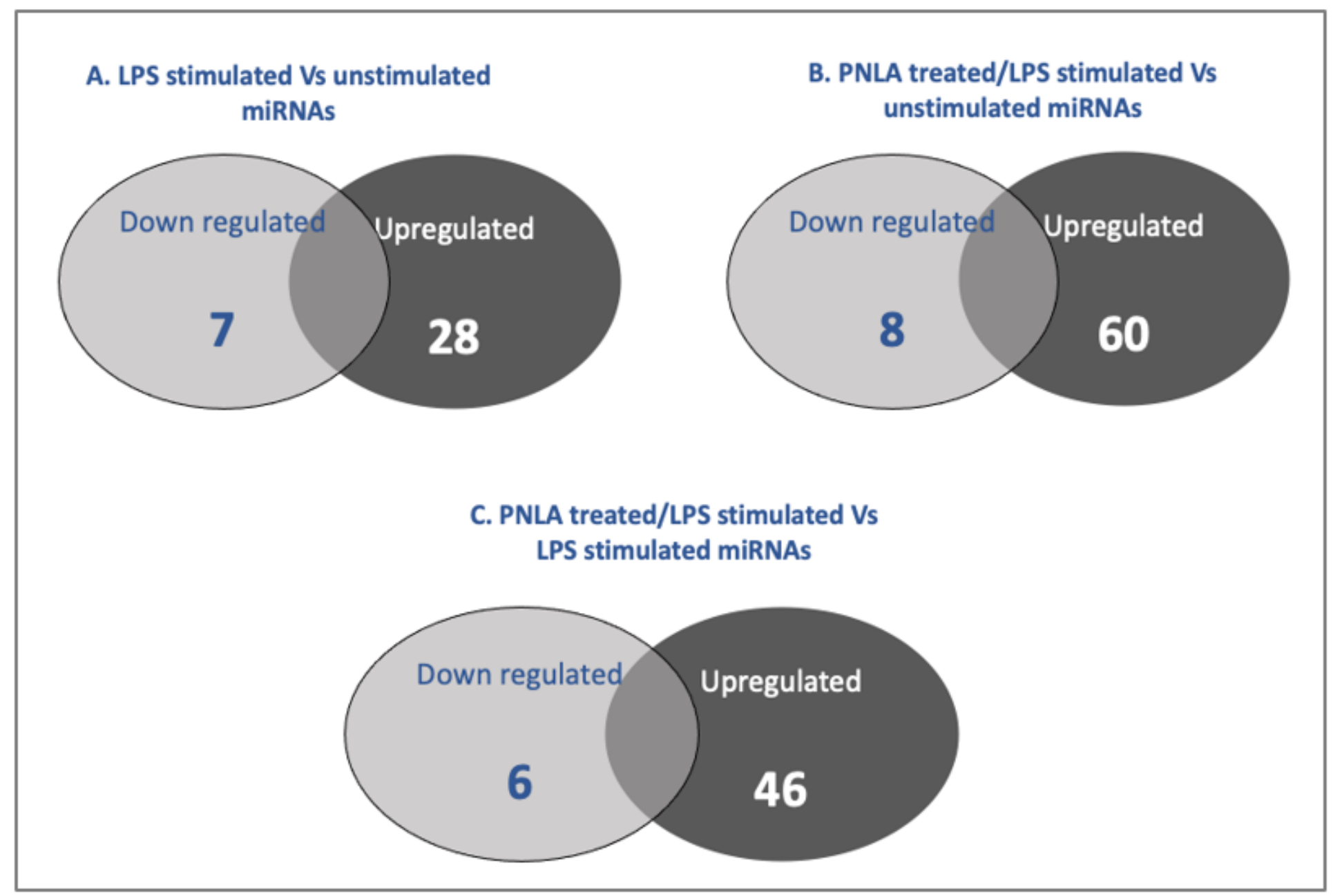

\section{Figure 4}

Venn diagrams showing the significantly affected miRNAs; (A) unstimulated (vehicle) vs LPS-stimulated monocytes (B) PNLA-treated LPS-stimulated vs vehicle-treated LPS-stimulated monocytes, and (C) PNLAtreated LPS-stimulated vs un- stimulated (vehicle-treated) monocytes ( $p$ value $<0.05$ ). 
A

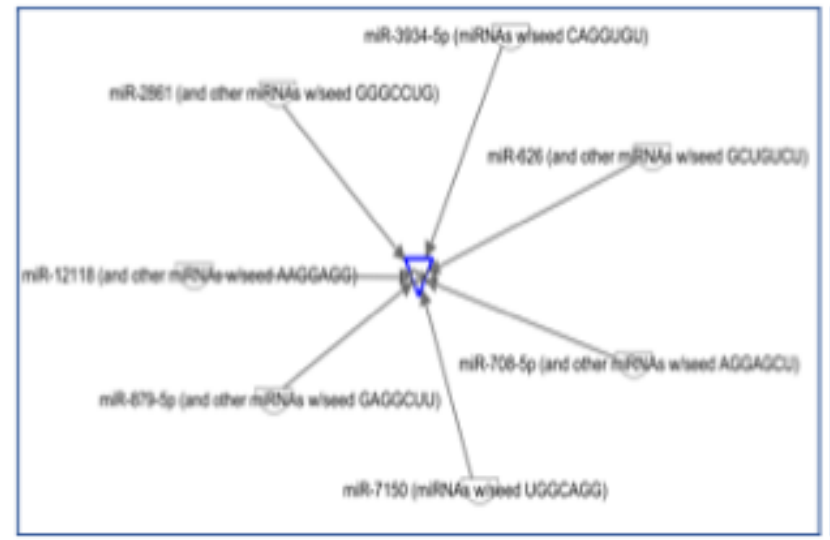

B

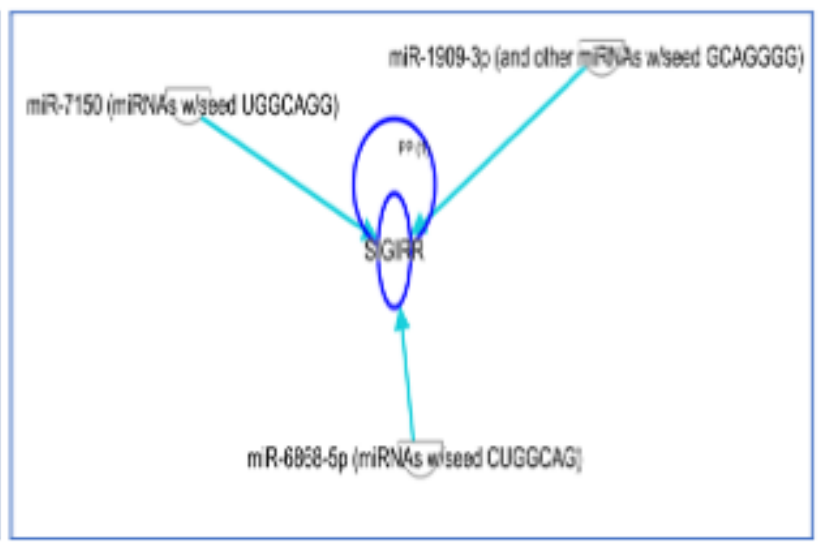

C

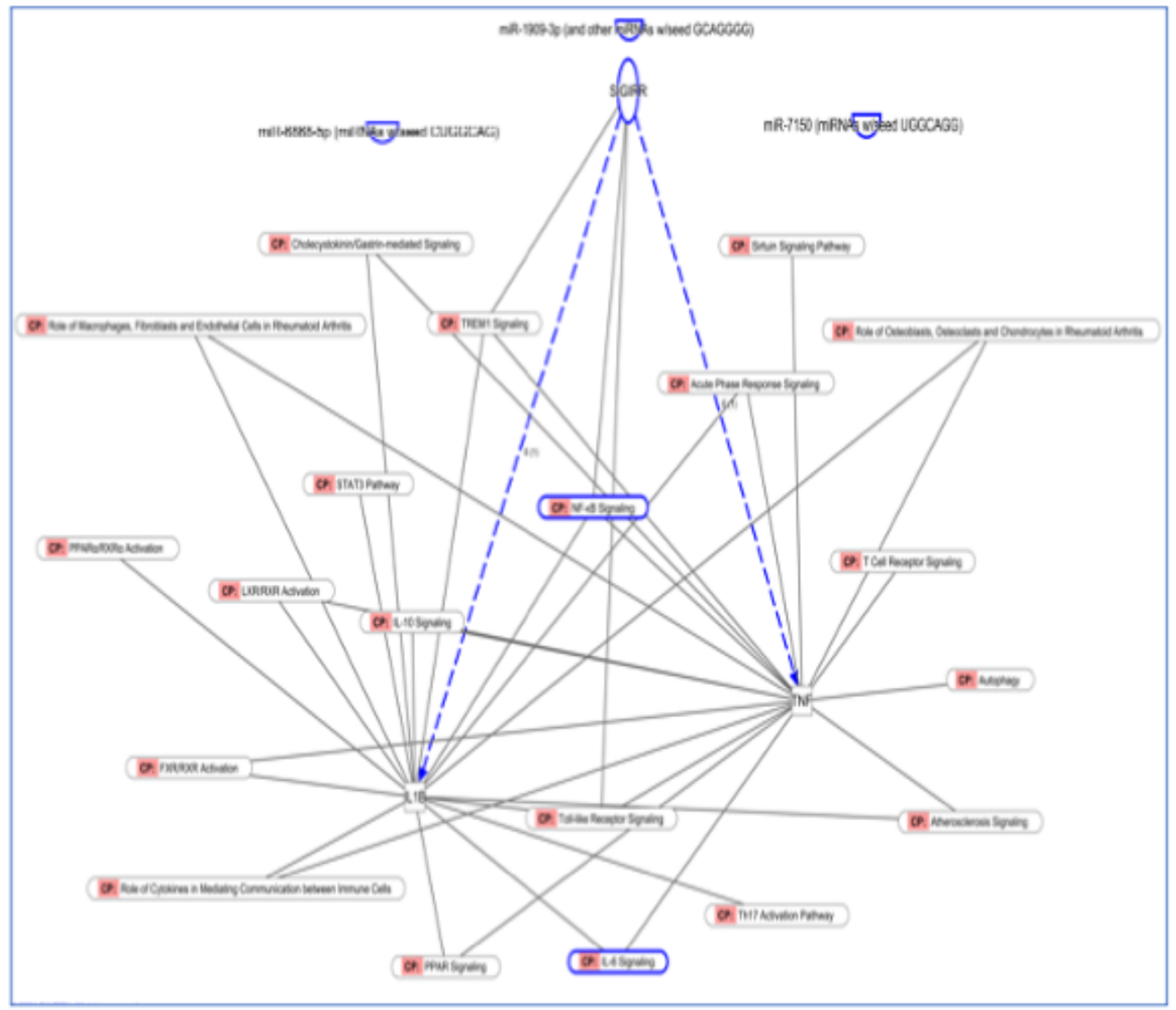

\section{Figure 5}

miRNAs targeted PDK4 and SIGIRR is downstream for miRNAs and upstream of metabolic and inflammatory pathways. (A) PDK4 mRNA is a target for miR-3173, miR-2861, miR-626, miR-28, miR-7150, miR-3188, miR-3934, and miR-1909. (B) SIGIRR mRNA is a target for miR-1909-3P, miR-7150, miR-68685P and (other miRNAs w/seed GCAGGGG) targeted SIGIRR mRNA. (C) SIGIRR is an upstream for many signalling pathways involved in regulation of transcription factors, inflammatory cytokines, and 
metabolic molecules. It negatively regulates NF-KB Signalling, TLR signalling, TREM1 signalling. CP; Canonical pathway.

\section{Supplementary Files}

This is a list of supplementary files associated with this preprint. Click to download.

- SupplementaryFigure1.pptx

- SupplementaryFigure2.pptx

- SupplementaryFigure3.pptx

- SupplementaryFigure4.pptx

- SupplementaryFigure5.pptx 